C. M. R. Santos · N. F. Martins · H. M. Hörberg

E. R. P. de Almeida $\cdot$ M. C. F. Coelho $\cdot$ R. C. Togawa

F. R. da Silva $\cdot$ A. R. Caetano $\cdot$ R. N. G. Miller

M. T. Souza Jr

\title{
Analysis of expressed sequence tags from Musa acuminata ssp. burmannicoides, var. Calcutta 4 (AA) leaves submitted to temperature stresses
}

Received: 29 June 2004 / Accepted: 7 March 2005/Published online: 20 April 2005

(C) Springer-Verlag 2005

\begin{abstract}
In order to discover genes expressed in leaves of Musa acuminata ssp. burmannicoides var. Calcutta 4 (AA), from plants submitted to temperature stress, we produced and characterized two full-length enriched cDNA libraries. Total RNA from plants subjected to temperatures ranging from $5^{\circ} \mathrm{C}$ to $25^{\circ} \mathrm{C}$ and from $25^{\circ} \mathrm{C}$ to $45^{\circ} \mathrm{C}$ was used to produce a COLD and a HOT cDNA library, respectively. We sequenced 1,440 clones from each library. Following quality analysis and vector trimming, we assembled 2,286 sequences from both libraries into 1,019 putative transcripts, consisting of 217 clusters and 802 singletons, which we denoted Musa acuminata assembled expressed sequence tagged (EST) sequences (MaAES). Of these MaAES, 22.87\% showed no matches with existing sequences in public databases. A global analysis of the MaAES data set indicated that $10 \%$ of the sequenced cDNAs are present in both cDNA libraries, while $42 \%$ and $48 \%$ are present only in the COLD or in the HOT libraries, respectively. Annotation of the MaAES data set categorized them into 22 functional classes. Of the 2,286 high-quality sequences, 715 $(31.28 \%)$ originated from full-length cDNA clones and resulted in a set of 149 genes.
\end{abstract}

Communicated by J.S. Heslop-Harrison

C. M. R. Santos · N. F. Martins · H. M. Hörberg

E. R. P. de Almeida - M. C. F. Coelho - R. C. Togawa

F. R. da Silva · A. R. Caetano - M. T. Souza Jr (ه)

Embrapa Genetic Resources and Biotechnology,

Caixa Postal 02372, Brasilia,

CEP 70.770-900, Distrito Federal, Brazil

E-mail: msouza@cenargen.embrapa.br

Tel.: + 55-61-4484722

Fax: + 55-61-3403624

R. N. G. Miller

Universidade Católica de Brasília, SGAN 916,

Módulo B, Brasilia, Distrito Federal,

CEP 70.790.160, Brazil

\section{Introduction}

Banana (Musa spp.) is cultivated in numerous tropical countries throughout the world, and in many of these countries its cultivation and marketing play very important roles, both economically and socially. In 2003, Brazil produced 6,469,470 Mton of bananas (approx. $9.5 \%$ of the world's production) in an area covering 507,000 ha (FAO 2004), making it the world's second largest producer of bananas. Bananas are considered to be a high-energy food and are also rich in minerals and vitamins. The fruit is cultivated throughout Brazil and is mainly traded in the domestic market. The majority of the banana farmers are small-scale producers with the crop grown principally as an supplementary source of income.

Banana is a monocotyledon and has a relatively small genome, $500-600 \mathrm{Mb}$ in its haploid state (only $25 \%$ larger than rice) distributed over 11 chromosomes (The Global Musa Genomics Consortium 2002). In 2001, the International Network for the Improvement of Banana and Plantain (INIBAP) launched the Global Musa Genomics Consortium (GMGC). This consortium is an initiative that aims to apply genomics to the sustainable improvement of banana and to develop freely accessible resources for Musa genomics using new knowledge and tools to enable both targeted conventional breeding and transgenic strategies. The GMGC strategy will also allow better utilization and maintenance of Musa biodiversity to ensure future food and income security for millions of people in developing countries.

In order to better understand the processes of plant development and their response to environmental stresses, it is necessary to identify and understand the function and regulation of the genes involved in the process. Developments in molecular biology have provided tools to enable insights into changes in the transcriptome that arise when a plant is submitted to 
different kinds of stresses. For the assessment of gene expression, methodologies such as large-scale singlepass sequencing of cDNA clones to generate expressed sequence tags (ESTs) can be utilized. ESTs provide a quantitative method to measure specific transcripts within a cDNA library and represent a powerful tool for gene discovery, gene expression, gene mapping and the generation of gene profiles (Rudd 2003).

Adverse conditions such as drought, salinity and temperature extremes are environmental factors that impose major limits on plant productivity. To overcome such limitations and improve production, breeders need to develop crops more tolerant to these stresses (Cushman and Bohnert 2000). Temperature stress in plants may affect physiological and biochemical processes (Grover et al. 2000), which are transduced through a chain of signaling molecules that ultimately affect regulatory elements of stress-inducible genes. As a consequence these environmental stresses, the synthesis of different proteins is initiated, including transcription factors, enzymes, molecular chaperons, ion channels and transporters involved in signaling cascades and transcriptional control (Mukhopadhyay et al. 2004). A network of interconnected cellular stress response is fundamental for plant survival.

Most fruit crops of tropical origin are sensitive to chilling temperatures, thereby limiting production. Chillsensitive tropical species, such as Musa spp., can be irreparably damaged when temperatures drop below $10^{\circ} \mathrm{C}$. Injuries are caused by impaired cellular processes including, among others, alterations in membrane properties, interactions between macromolecules and enzymatic reactions, protein denaturation, change in hormone levels, increases in insoluble sugars and the production of reactive oxygen species (Xiong et al. 2002).

Conversely, stress caused by high temperatures induces grana disintegration, reduces electron transport, affects energy distribution, changes the structural organization of the thylakoid membranes, the membrane fluidity and the ion balance and decreases the activity of Rubisco 1,5 biphosphate carboxylase, thereby limiting photosynthesis (Grover et al. 2000).

We describe the isolation, sequencing, clustering and annotation of 2,880 ESTs generated from two cDNA libraries that were constructed from Musa acuminata ssp. burmannicoides var. Calcutta 4 (AA) leaves subjected to different temperature stresses.

\section{Materials and methods}

In vitro-micropropagated Musa acuminata ssp. burmannicoides var. Calcutta 4 (AA) plants, 30-cm high, were used in this study. These plants were obtained from the Banana Germplasm Bank at Embrapa Cassava and Tropical Fruits, Cruz das Almas, Bahia, Brazil [Musa Germplasm Information System (MGIS) accession no. ITC0249]. Prior to applying the temperature stress conditions, we maintained the banana plants for $48 \mathrm{~h}$ in an environment chamber at $25^{\circ} \mathrm{C}$ and $80 \%$ air humidity under a 16/8-h (light/dark) photoperiod.

For the construction of the HOT cDNA library, leaf samples were first removed from plants at $25^{\circ} \mathrm{C}(\mathrm{H} 0)$ then, following a temperature rise to $35^{\circ} \mathrm{C}$, samples were collected after $1 \mathrm{~h}(\mathrm{H} 1), 2 \mathrm{~h}(\mathrm{H} 2)$ and $3 \mathrm{~h}(\mathrm{H} 3)$. The temperature was then increased to $45^{\circ} \mathrm{C}$, and new samples were collected after $1 \mathrm{~h}(\mathrm{H} 4), 2 \mathrm{~h}(\mathrm{H} 5), 3 \mathrm{~h}(\mathrm{H} 6), 9 \mathrm{~h}$ $(\mathrm{H} 7)$ and $21 \mathrm{~h}(\mathrm{H} 8)$ following the change in temperature.

For the construction of the COLD cDNA library, leaf samples were first collected from plants at $25^{\circ} \mathrm{C}(\mathrm{C} 0)$ then, with the temperature decreased to $15^{\circ} \mathrm{C}$, samples were collected $1 \mathrm{~h}(\mathrm{C} 1), 2 \mathrm{~h}(\mathrm{C} 2)$ and $3 \mathrm{~h}(\mathrm{C} 3)$ following the temperature change. The temperature was then decreased to $5^{\circ} \mathrm{C}$, and new samples were collected after $1 \mathrm{~h}$ (C4), $2 \mathrm{~h}$ (C5), $3 \mathrm{~h}$ (C6), $9 \mathrm{~h}$ (C7) and $21 \mathrm{~h}$ (C8) following the change in temperature. The collected leaf tissues were immediately frozen in liquid $\mathrm{N}$ and stored at $-80^{\circ} \mathrm{C}$ until total RNA extraction.

Total RNA was isolated using the Plant RNA Reagent kit (Invitrogen Life Technologies, Carlsbad, Calif.), according to the protocols provided by the manufacturer. For each library, total RNA preparations from the eight time-point samples were combined in equivalent quantities. The resulting bulked sample (1 mg of total RNA) was then submitted to poly(A) + RNA purification using the FastTrack 2.0 mRNA Isolation kit (Invitrogen Life Technologies) according to manufacturer's instructions. Full-length cDNA enriched, non-normalized, cDNA libraries were constructed with the Creator Smart cDNA library kit and cloned into the pDNR-LIB vector (Clontech Laboratories, Palo Alta, Calif.).

The $5^{\prime}$ ends of the cDNA clones were sequenced with the Embrapa Genetic Resources and Biotechnology's DNA sequencing platform using the M13 forward primer (5'-TGT AAA ACG ACG GCC AGT-3') and Dye Terminator chemistry on automated sequencers. Trace files were submitted to the GENOMA system at the Embrapa Genetic Resources and Biotechnology Bioinformatics Laboratory, where they were stored in the MUSA ESTs database until sequence processing and analysis.

Base calling and quality assignment of individual bases of the EST sequences were performed using the program PHRED (Ewing et al. 1998). Ribosomal poly(A) tails, low-quality sequences and vector and adapter regions were removed as described by Telles and da Silva (2001) with minor adaptations. The resulting sets of cleaned sequences were assembled into clusters of overlapping sequences using the CAP3 assembler (Huang and Madan 1999), with individual base quality and default parameters. The resulting contigs and singlets were designated Musa acuminata assembled EST sequences (MaAES), and similarities between these and sequences in the GenBank nr (Benson et al. 2002) database were retrieved using BLASTX (e value $>10^{-3}$ ) (Altschul et al. 1997). 


\section{Results}

A total of 1,440 ESTs from each of the two cDNA libraries were sequenced. All 2,880 reads sequenced showed an average length of 891 bases and an average of 581 bases with PHRED $>20 ; 89.3 \%$ of the total reads had more than 350 bases with PHRED $>20$. The read-cleaning procedure discarded 594 reads, maintaining 1,143 sequences from the COLD library and 1,143 from the HOT library. The 2,286 high-quality EST sequences were then grouped into 1,019 MaAES (44.6\% novelty). A total of 217 MaAES were formed by two or more reads and contained 1,484 ESTs. The remaining 802 MaAES consisted of single EST sequences. Of the 217 contigs, 47 were derived from ESTs from the COLD library, 73 from ESTs from the HOT library, while 97 contained ESTs from both libraries. Among the singlets, 376 originated from the COLD library and 426 from the HOT library (Fig. 1). The frequency of EST distribution after clustering is shown in Fig. 2. Five MaAES had 50 or more ESTs, with the largest containing 240 ESTs. The most frequent groups of MaAES $(42.21 \%$ or $965 \mathrm{Ma}-$ AES) contained one to five ESTs.

Identification of putative genes of the 1,019 MaAES was carried out initially by a similarity search using
BLASTX comparisons against the Genbank $\mathrm{nr}$ database (BLASTX 2.2.3). Two hundred and thirty-three MaAES, or $22.87 \%$, had no evident homology with any protein sequence in the database. Ten sequences produced matches with known Musa ssp. proteins contained in this public database. The 1,019 transcripts encode 535 proteins with different assigned functions. The two most abundant MaAES contained 240 and 90 transcripts, with homology to the ribulose bisphosphate carboxylase small-chain and photosystem II (PSII) $10-\mathrm{kDa}$ protein, respectively (Table 1 ). This result was expected since we used plant leaf material used to construct the cDNA libraries. In addition, a series of proteins known to be involved in response to stresses were also present in the group of most abundant MaAES, including class-I lowmolecular-weight (LMW) heat-shock protein, metallothionein-like protein, carbonic anhydrase and rubisco activase.

In order to predict functions for the proteins encoded by the expressed genes sequenced, we carried out a search on all 1,019 MaAES in the eukaryotic orthologous groups (KOG) (Tatusov et al. 2003), which contain groups of orthologous proteins of seven eukaryotic genomes. This comparison resulted in 502 MaAES with no evident homologies with sequences in the database, and 517 MaAES homologous to proteins with known

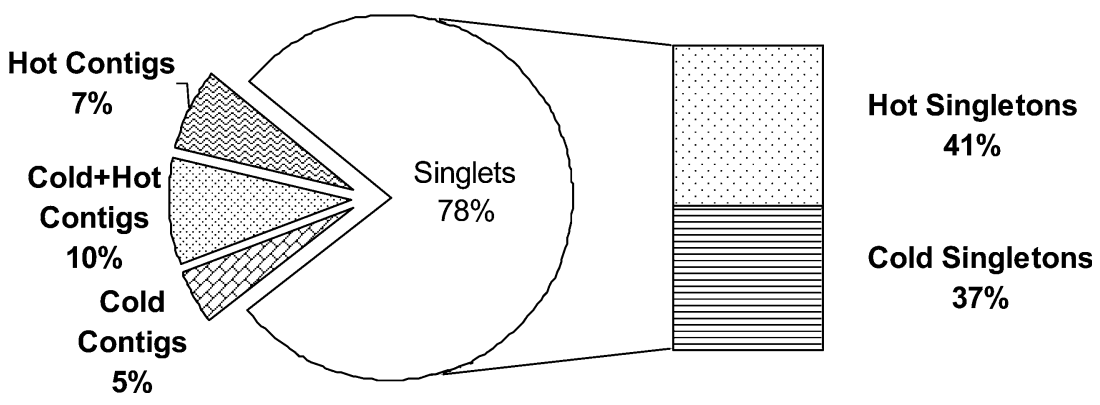

Fig. 1 Distribution and frequency of Musa acuminata assembled EST sequences (MaAES) following the clustering of 2,286 highquality EST sequences with the CAP3 algorithm. ESTs were obtained from two M. acuminata var. Calcutta 4 (AA) leaf cDNA libraries:
COLD - plants subjected to temperatures ranging from $5^{\circ} \mathrm{C}$ to $25^{\circ} \mathrm{C}$ - and HOT - plants submitted to temperatures ranging from $25^{\circ} \mathrm{C}$ to $45^{\circ} \mathrm{C}$
Fig. 2 Distribution and number of clustered sequences. The frequency of EST distribution was obtained following CAP3 analysis. All sequences with at least 100 nucleotides having a PHRED $\geq 20$ were considered for clustering. Most clusters $(78.7 \%$ ) consist of singlets, while the largest cluster comprised 240 ESTs

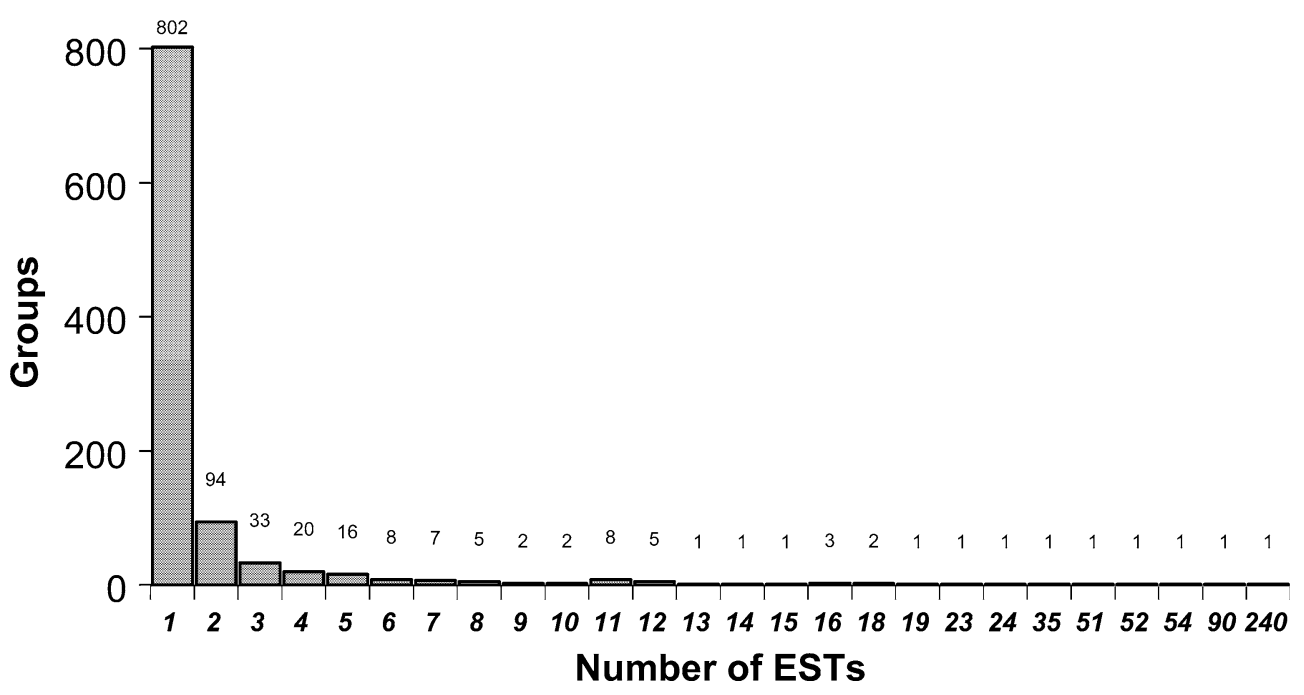


Table 1 Summary of the most abundant similarities between MaAES and sequences of GenBank nr database sequences

\begin{tabular}{|c|c|}
\hline $\begin{array}{l}\text { Number } \\
\text { of ESTs }\end{array}$ & Gene annotation \\
\hline 12 & $\begin{array}{l}\text { ref } \mid \text { NP_175963.1| photosystem I subunit V precursor-related } \\
\text { (Arabidopsis thaliana) }\end{array}$ \\
\hline 14 & $\mathrm{gb} \mid$ AAO49652.1| photosystem I-N subunit (Phaseolus vulgaris) \\
\hline 15 & pir T12245 ABA stress ripening protein - common ice plant \\
\hline 16 & $\begin{array}{l}\text { pir|S17446 photosystem II oxygen-evolving complex protein } \\
2 \text { precursor-common tobacco }\end{array}$ \\
\hline 16 & $\begin{array}{l}\text { sp } \mid \text { P16016 } \mid \text { CAHC_SPIOL Carbonic anhydrase, chloroplast precursor } \\
\text { (carbonate dehydratase) }\end{array}$ \\
\hline 18 & $\mathrm{gb} \mid$ AAM97011.1| expressed protein (Arabidopsis thaliana) \\
\hline 18 & $\mathrm{gb} \mid$ AAM28293.1| class-1 LMW heat shock protein (Ananas comosus) \\
\hline 19 & gb AAR91119.1 chloroplast hypothetical protein (Zea mays) \\
\hline 23 & gb|AAK25801.1| rubisco activase [Zantedeschia aethiopica] \\
\hline 24 & $\mathrm{gb} \mid$ AAD 41409.1 | cytosolic class II LMW heat-shock protein (Prunus dulcis) \\
\hline 35 & sp|P30222|HS2C_PETHY Small heat-shock protein, chloroplast precursor \\
\hline 52 & $\mathrm{emb}|\mathrm{CAB} 52585.1|$ metallothionein-like protein (Elaeis guineensis) \\
\hline 54 & emb $\mid$ CAB85630.1 $\mid$ putative metallothionein-like protein (Vitis vinifera) \\
\hline 90 & gb|AAN60205.1 photosystem II 10-kDa protein(Xerophyta humilis) \\
\hline 240 & $\begin{array}{l}\text { sp }|\mathrm{O} 24045| \mathrm{RBS} \text { MUSAC ribulose bisphosphate carboxylase small-chain, } \\
\text { chloroplast precursor (RuBisCo small subunit) }\end{array}$ \\
\hline
\end{tabular}

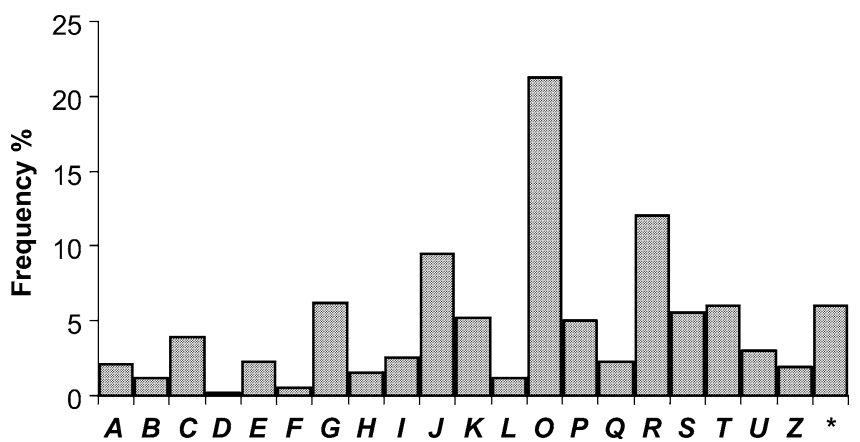

Fig. 3 Functional classification and class frequency of M. acuminata assembled EST sequences (MaAES) from the two cDNA libraries, according to eukaryotic clusters of orthologs (KOG). Designations of functional categories: $A$ RNA processing and modification, $B$ chromatin structure and dynamics, $C$ energy production and conversion, $D$ cell-cycle control and mitosis, $E$ amino acid metabolism and transport, $F$ nucleotide metabolism and transport, $G$ carbohydrate metabolism and transport, $H$ coenzyme metabolism, $I$ lipid metabolism, $J$ translation, $K$ transcription, $L$ replication and repair, $O$ post-translational modification, protein turnover, chaperone functions, $P$ inorganic ion transport and metabolism, $Q$ secondary metabolites biosynthesis, transport and catabolism, $R$ general functional prediction only, $S$ function unknown, $T$ signal transduction, $U$ intracellular trafficking and secretion, $Z$ cytoskeleton, * non-conclusive

functions. These latter MaAES were categorized into 22 functional classes, of which $21.3 \%$ corresponded to genes involved in post-translational modification, protein turnover and chaperone functions, $12 \%$ to genes with general functional prediction only, $9.5 \%$ to genes involved in translation, $6.2 \%$ to genes related to carbohydrate metabolism and transport and $6 \%$ to genes involved in signal transduction (Fig. 3).

To assess the frequency of full-length transcripts in the cDNA libraries, we selected the 1,023 reads out of the 2,286 high-quality sequences $(44.8 \%)$ obtained that showed close homologies in the SwissProt database (BLASTX e value lower than $10^{-30}$ ), and searched for the presence of the first 15 amino acids of the protein in the query. A total of 715 of the 1,023 reads $(69.8 \%)$ fell in this category and were considered to be full-length inserts. These 715 ESTs corresponded to 149 genes. Most of these genes from the full-length cDNA clones that were identified were classified as having photosynthesis or chaperone functions, for example: ribulose bisphosphate carboxylase small-chain, chlorophyll $a / b$, PS I and PS II, small heat-shock protein (sHSP) and ribulose bisphosphate carboxilase/oxygenase activase (Table 1).

\section{Discussion}

A total of 1,019 MaAES were generated from two cDNA libraries isolated from banana plant leaves submitted to temperature stresses. Our analysis of these MaAES has provided us with a new insight into the Musa genome in relation to low and high temperature stresses, an abiotic stress which bananas are commonly exposed to in many production areas worldwide. Most of the MaAES identified in the cDNA libraries we characterized (Fig. 3) fall within three functional and frequency classes: class A, post-translational modification, protein turnover, chaperone functions; class B, general functional prediction category; class $\mathrm{C}$, translation. Class A accounts for genes involved in the response of the plant to environmental stresses, while class B, the second most frequent functional class, represents housekeeping genes. Class $\mathrm{C}$ includes genes involved in translation. Together, these three groups represent approximately $43 \%$ of the MaAES identified.

The development and survival of plants is constantly challenged by changes in environmental conditions. To overcome adverse conditions, plants elicit complex physiological and molecular responses, activating a large set of genes leading to the accumulation of specific 
stress-associated proteins, such as HSPs (Borges et al. 2001; Sanmiya et al. 2004). Although there is a general decrease in the synthesis of many constitutively expressed proteins in a stressed situation, the translation machinery remains active and the synthesis of several HSPs is enhanced. About $30 \%$ of the MaAES we identified are genes involved in the response to environmental stresses and in translation.

HSPs and molecular chaperones function in the stabilization of proteins and membranes and in assisting protein refolding under stress conditions. The induction of HSP synthesis commonly occurs immediately after the plant has been exposed to an elevated temperature (Gusev et al. 2002). The small heat-shock proteins (sHSPs) range in size from $12 \mathrm{kDa}$ to $40 \mathrm{kDa}$ (Vierling 1991) and are found to be the most prevalent in plants exposed to abiotic stresses, such as water, salt, oxidative stress and cold (Hamilton and Heckathorn 2001). Plants typically produce more than ten different types of sHSPs that belong to five distinct gene classes encoding proteins targeted to different cell compartments, such as the chloroplasts, cytoplasm, nucleus, mitochondria and endoplasmic reticulum (ER) (Gusev et al. 2002).

Chloroplast-located sHSPs have been implicated in the protection of the oxygen-evolving complex and electron transport of PS II against heat damage (Downs et al. 1999). Cytosolic sHSPs exist as large oligomers (approximately $200-800 \mathrm{kDa}$ ) composed solely or primarily of sHSPs. In Oryza sativa and Lycopersicon esculentum, the sHSPs OsHSP26 and HSP22 are induced by $\mathrm{H}_{2} \mathrm{O}_{2}$ (Lee et al. 2000). Plant cytosolic HSPs can form complexes containing mostly sHSP, but also HSP70 and RNA. These complexes are supposed to function as transient storage for non-heat-shock mRNA, thereby preventing their degradation during heat stress (Gusev et al. 2002). The mitochondrial HSP70 mediates protein transport across the inner membrane and protein folding in the matrix. The ER is a key compartment in cells that are specialized for protein export and contains many chaperones. The concentration of ER HSP70 is increased by elevated levels of misfolded proteins in the ER (Fink 1999).

A set of 27 cDNAs coding for HSPs was identified in this study, based on similarity searches against GenBank. Thirteen of these were sHSPs genes whose proteins are located in the cytoplasm. Twelve full-length cDNAs of HSPs were identified in this study, nine of which are of the sHSP family type and localized in the cytoplasm and chloroplast.

Several transcripts were widely expressed in both libraries, including the so-called housekeeping genes. Among the 2,286 sequenced transcripts were genes involved with the photosynthesis apparatus and those related to temperature stress, such as ribulose 1,5 biphosphate carboxylase/oxygenase (10.72\%), sHSP $(8.50 \%)$, HSP $(1.71 \%)$, PS I/II $(9.75 \%)$ and metallothionein-like protein $(6.91 \%)$. Other proteins, including rubisco activase, chlorophyll $a / b$-binding protein, early light-inducible protein, ubiquitin, cytochrome $c$, ferre- doxin, calmodulin and abscisic acid (ABA) stress protein, were also present, representing less than $2 \%$ of clones in the libraries. We have identified three full-length sequences of ribulose 1,5 bisphosphate carboxylase/oxygenase, nine of PS I/II and one of metallothionein-like protein.

A number of plant species increase their cellular levels of the phytohormone ABA when subjected to low temperatures (Thomashow 1999). Several studies have suggested that ABA has a role in inducing the expression of genes related to activating the cold-acclimation response (Thomashow 1999). Antifreeze proteins induced by ABA were found in somatic embryos of Picea abies and cell suspensions of Rhodiola algida ( $\mathrm{Lu}$ et al. 2000). We identified two different Musa ssp. genes related to the ABA response to cold acclimation.

Plants submitted to stress resulting from cold environments show diverse changes at the cellular and molecular levels. These changes include: (1) modifications in the composition of the plasma membranes (Uemura et al. 1995); (2) intracellular accumulation of compatible osmolytes, such as soluble sugars, prolines and betaines (Hare et al. 1998), HSPs (Ukaji et al. 1999) and cold-regulated (COR) proteins (Thomashow 1999); (3) extracellular accumulation of antifreeze proteins (Griffith and Antikainen 1996); (4) changes in the properties of the cell walls (Fujikawa and Kuroda 2000). These changes have been found to be associated with increased freezing tolerance (Thomashow 1999). Many of these changes are regulated through qualitative and quantitative modifications of gene expression, leading to the accumulation of newly synthesized proteins and mRNAs. One low-temperature responsive gene was identified in the present study.

One important step in the control of stress responses appears to be the transcriptional activation or repression of genes. Many genes induced by stress, including those encoding transcription factors, have been identified in this study, including Myb, zinc finger and heat-shock transcription factor. Some of these are known to be essential for stress tolerance (Nagaoka and Takano 2003; Lohmann et al. 2004; Rizhsky et al. 2004).

When we accessed the National Center of Biotechnology Information database in early June 2004, we found 651 nucleotide sequences and 227 protein sequences from $M$. acuminata. The present study, which developed and characterized two cDNA libraries of M. acuminata ssp. burmannicoides var. Calcutta 4 (AA), identified 535 unigenes, with 149 from full-length cDNA clones.

The data generated in this study contribute largely to the Musa Genomics data set and provide an important resource for understanding the biology of this plant. The data also provide new targets for the genetic engineering of banana and other crops for improved tolerance and resistance to abiotic stresses. In addition, our results may facilitate genomic analyses in other plant genera, gene discovery as well as functional characterization and annotation of genomic sequences. 
All of the data generated in this study will be available for public access at Embrapa Genetic Research and Biotechnology homepage (http://www.cenargen.embrapa.br/index.html). These data form part of the DATA Musa database (a genome database for Musa spp. consisting of Musa spp. DNA sequences and Sequence Information), which is maintained by Embrapa and open to public access following the signing of a letter of agreement. Those interested in accessing DATA_Musa should contact our laboratory through the Musa Genomics Brazil homepage (http://genoma.embrapa.br/ musa/en/index.html).

Acknowledgements C.M.R.S. and H.M.H. were supported by fellowships from the Brazilian National Council for Scientific and Technological Development (CNPq). The investigation reported here forms part of the ongoing investigations in the research project "Análise da Estrutura Primária do Genoma A de Musa acuminata"-Processo institucional 680.398/01-5, sponsored by CNPq. This work represents a part of the Brazilian participation in the Global Musa Genomics Consortium (http://www.inibap.org).

\section{References}

Altschul SF, Madden TL, Schaffer AA, Zhang J, Zhang Z, Miller W, Lipman DJ (1997) Gapped BLAST and PSI-BLAST: a new generation of protein database search programs. Nucleic Acids Res 25:3389-3402

Benson DA, Karsch-Mizrachi I, Lipman DJ, Ostell J, Rapp BA, Wheeler DL (2002) GenBank. Nucleic Acids Res 30:17-20

Borges JC, Peroto MC, Ramos CHI (2001) Molecular chaperone genes in the sugarcane expressed sequence database (SUCEST). Genet Mol Biol 24:85-92

Cushman JC, Bohnert HJ (2000) Genomic approaches to plant stress tolerance. Curr Opin Plant Biol 3:117-124

Downs CA, Ryan SL, Heckathorn SA (1999) The chloroplast small heat-shock protein: evidence for a general role in protecting photosystem II against oxidative stress and photoinhibition. J Plant Physiol 155:488-496

Ewing B, Hillier L, Wendl MC, Green P (1998) Base-calling of automated sequencer traces using phred. I. Accuracy assessment. Genome Res 8:175-185

FAO (2004) http://apps.fao.org

Fink AL (1999) Chaperone-mediated protein folding. Physiol Rev 79:425-449

Fujikawa S, Kuroda K (2000) Cryo-scanning electron microscopic study on freezing behavior of xylem ray parenchyma cells in hardwood species. Micron 31:669-686

Griffith M, Antikainen M (1996) Extracellular ice formation in freezing-tolerant plants. Adv Low-Temp Biol 3:107-139

Grover A, Agarwal M, Katiyar-Agarwal S, Sahi C, Agarwal S (2000) Production of high temperature tolerant transgenic plants through manipulation of membrane lipids. Curr Sci 79:557-559

Gusev NB, Bogatcheva NV, Marston SB (2002) Structure and properties of small heat shock protein (sHSP) and their interaction with cytoskeleton proteins. Biochemistry 67:511-519
Hamilton EW, Heckathorn SA (2001) Mitochondrial adaptations to sodium chloride: complex I is protected by anti-oxidants and small heat-shock proteins, while Complex II is protected by proline and betaine. Plant Physiol 126:166-174

Hare PD, Cress WA, Van Staden J (1998) Dissecting the roles of osmolyte accumulation during stress. Plant Cell Environ 21:535-553

Huang X, Madan A (1999) CAP3: a DNA sequence assembly program. Genome Res 9:868-877

Lee BH, Won SH, Lee HS, Miyao M, Chubg WI, Kim IJ, Jo J (2000) Expression of the chloroplast localized small heat-shock protein by oxidative stress in rice. Gene 245:283-290

Lohmann C, Eggers-Schumacher G, Wunderlich M, Schoffl F (2004) Two different heat shock transcription factors regulate immediate early expression of stress genes in Arabidopsis. Mol Genet Genomics 271:11-21

Lu CF, Jian LC, TY Kuang (2000) Secretory antifreeze proteins produced in suspension culture cells of Rhodiola algida var. tangutica during cold acclimation. Prog Biochem Biophys 27:555-559

Mukhopadhyay A, Vij S, Tyagi AK (2004) Overexpression of a zinc-finger protein gene from rice confers tolerance to cold, dehydration, and salt stress in transgenic tobacco. Proc Natl Acad Sci USA 101:6309-6314

Nagaoka S, Takano T (2003) Salt tolerance-related protein STO binds to a Myb transcription factor homologue and confers salt tolerance in Arabidopsis. J Exp Bot 54:2231-2237

Rizhsky L, Davletova S, Liang H, Mittler R (2004) The zinc finger protein Zat12 is required for cytosolic ascorbate peroxidase 1 expression during oxidative stress in Arabidopsis. J Biol Chem 279:11736-11743

Rudd S (2003) Expressed sequence tags: alternative or complement to whole genome sequences? Trends Plant Sci 8:321-329

Sanmiya K, Suzuki K, Egawa Y, Shono M (2004) Mitochondrial small heat-shock protein enhances thermotolerance in tobacco plants. FEBS Lett 557:265-268

Tatusov RL, Fedorova ND, Jackson JD, Jacobs AR, Kiryutin B, Koonin EV, Krylov DM, Mazumder R, Mekhedov SL, Nikolskaya AN, Rao BS, Smirnov S, Sverdlov AV, Vasudevan S, Wolf YI, Yin JJ, Natale DA (2003) The COG database: an updated version includes eukaryotes. Bioinformatics 4:1-14

Telles GP, da Silva FL (2001) Trimming and clustering sugarcane ESTs. Genet Mol Biol 24:17-23

The Global Musa Genomics Consortium (2002) A strategy for the Global Musa Genomics Consortium. Report of a meeting held in Arlington, USA 17-20 July 2001. International Network for the Improvement of Banana and Plantain, Montpellier, France

Thomashow MF (1999) Plant cold acclimation: freezing tolerance genes and regulatory mechanisms. Annu Rev Plant Physiol Plant Mol Biol 50:571-599

Uemura M, Joseph RA, Steponkus PL (1995) Cold acclimation of Arabidopsis thaliana: effect on plasma membrane lipid composition and freeze-induced lesions. Plant Physiol 109:15-30

Ukaji N, Kuwabara C, Takezawa D, Arakawa K, Yoshida S, Fujikawa S (1999) Accumulation of small heat-shock protein homologs in the endoplasmic reticulum of cortical parenchyma cells in mulberry in association with seasonal cold acclimation. Plant Physiol 120:481-489

Vierling E (1991) The roles of heat shock proteins in plants. Annu Rev Plant Physiol Plant Mol Biol 42:579-620

Xiong L, Schumaker KS, Zhu JK (2002) Cell signaling during cold, drought, and salt stress. Plant Cell 14:S165-S183 